\title{
Sarcoma de aplicação felino
}

\section{Feline injection-site sarcoma}

\author{
Carolina Scarpa Carneiro ${ }^{1 *}$; Genilson Fernandes Queiroz ${ }^{2}$; \\ Maria Beatriz Cattony Zerwes ${ }^{3}$; Julia Maria Matera ${ }^{4}$
}

\section{Resumo}

O sarcoma de aplicação felino (SAF) é atualmente um grande desafio para o médico veterinário e também para o proprietário do felino acometido. Aplicações injetáveis por via subcutânea ou intramuscular, como vacinas e medicações, aparecem como iniciadoras do processo de neogênese dessa neoplasia, mais precisamente a inflamação persistente, causada pela lesão ao tecido sadio decorrente do fármaco ou antígeno administrado. Geralmente o SAF apresenta comportamento mais agressivo quando comparado ao sarcoma não associado à aplicação. $O$ tratamento mais eficaz ainda não está estabelecido, mas acredita-se que a multimodalidade de terapias, cirurgia, radioterapia e quimioterapia seja a opção mais indicada. O conhecimento da afecção em todos os seus aspectos irá fornecer aos colegas profissionais subsídios em relação a melhor maneira de abordá-la em termos de diagnóstico, tratamento e prevenção.

Palavras-chave: Neoplasia, sarcoma, tumor, felino, vacinas, medicamentos

\begin{abstract}
The feline injection-site sarcoma (FIS) is a challenge for the veterinarian and the affected cat's owner. The injectable applications (vaccines, medications) seems to be the reason for that neoplasia, more specifically, the inflammation caused by injury of given drugs or antigens to the health tissue. Generally the FIS presents a more aggressive behavior when compared to sarcoma not associated to application. The most effective treatment has not been established yet, but it is believed that a multimodality of therapies, surgery, radiotherapy, and chemotherapy would be the most indicated option. The knowledge of the illness in all of its aspects will supply to professionals colleges subsidies in relation to the best way to approach its diagnosis and treatment.
\end{abstract}

Key words: Neoplasia, sarcoma, tumor, feline, vaccines, drugs

1 Mestre em Clínica Cirúrgica Veterinária - Faculdade de Medicina Veterinária e Zootecnia (FMVZ), Universidade de São Paulo (USP). Av. Prof. Orlando Marques de Paiva, 87, 05508-900, Cidade Universitária, São Paulo, SP, Brasil. E-mail: sccarolin@usp. br.

2 Doutorando do Curso de Pós-Graduação do Departamento de Cirurgia FMVZ/USP, São Paulo, SP, Brasil;

3 Médica Veterinária - Mestre em Clínica Cirúrgica Veterinária, Vargem Grande Paulista - SP;

4 Professora Titular do Departamento de Cirurgia FMVZ/USP, São Paulo, SP, Brasil.

* Autor para correspondência 


\section{Introdução}

Na década de 1980 dois eventos importantes aconteciam na Pensilvânia; o lançamento de vacinas inativadas com adjuvante a base de alumínio, em particular, a anti-rábica e a anti-vírus da leucemia felina (FeLV), e a publicação do estatuto que obrigava a vacinação anti-rábica para todos os gatos da Pensilvânia (KASS et al, 1993; HAUCK, 2003). Em meados de 1987, foi observado no Serviço de Patologia Cirúrgica da Universidade da Pensilvânia um aumento na freqüência (61\%) de reações inflamatórias naquelas amostras biopsiadas em locais que coincidiam com aplicação prévia de vacinas em cães e gatos. Foram enviados questionários aos veterinários, confirmando que estas reações ocorriam em sítios de prévia administração da vacina. A distribuição desses tumores coincidia com os locais comumente utilizados para vacinação, especificamente nas regiões interescapular, cervical e 1/3 médio femoral caudal de membros pélvicos, os quais diferem de regiões previamente relatadas para o surgimento de sarcomas (cabeça, membros e flanco) (HENDRICK; GOLDSCHMIDT, 1991).

O primeiro relato da evidência epidemiológica da correlação entre os sarcomas e os locais de administração de injeções foi então publicado, por Hendrick e Goldschmidt (1991). Autores demonstraram que havia correlação entre a administração de vacinas inativadas, contra leucemia felina e raiva principalmente, e o subseqüente desenvolvimento de sarcomas, com aumento da incidência quando mais de uma aplicação era administrada no mesmo local (SÉGUIN, 2002; POIRIER et al., 2002; HAUCK, 2003).

Apesar da correlação entre o aumento de casos de sarcomas em felinos e da aplicação de vacinas, a incidência e etiologia desses sarcomas permanecem desconhecidas (SÉGUIN, 2002). Atualmente, acredita-se que as vacinas anti-rábica e contra FeLV não são os únicos agentes capazes de causar o desenvolvimento de sarcomas nos locais de aplicação, mas qualquer agente que produza inflamação local exacerbada apresenta esse potencial etiológico (HENDRICK et al., 1994; SÉGUIN, 2002). Esta observação condiz com relatos de desenvolvimento de sarcomas em gatos após outros tipos de lesão inflamatória, como: no trauma ocular ou uveíte crônica (DUBIELZIG, 1984); na administração de lufenuron, acetato de metil predinisolona, penicilina de longa ação (KASS et al., 1993) ou de vacinas contra panleucopenia e rinotraqueite em felinos (SÉGUIN, 2002); bem como, em locais de traumatismos constantes por fio cirúrgico não absorvível (BURACCO et al., 2002).

Em 2003, BREARLEY publicou uma carta onde chamou a atenção para a preocupação dos proprietários em vacinar os animais, já que a possibilidade do desenvolvimento do sarcoma estava ligado inicialmente às aplicações vacinais. Por esse motivo sugere a nova nomenclatura para o sarcoma vacinal felino, "sarcoma no local da aplicação" ou "sarcoma de aplicação", já que também a etiopatogenia desse tipo tumoral e o papel da vacina no seu desenvolvimento, ainda não foram elucidados.

Mais recentemente, Kirpensteijn (2006) cita três razões para estudar o papel das aplicações na indução dos sarcomas: primeiro que algumas aplicações parecem causar uma reação inflamatória local considerável; segundo os sarcomas de aplicação são um grupo distinto histopatologicamente dos sarcomatas e ocorrem com maior freqüência que os sarcomas não relacionados às aplicações; e por último a localização desses sarcomas é muito sugestiva de que estes provenham de uma aplicação. O sarcoma pós-vacinal também já foi descrito em cães, sendo menos comum nessa espécie (VASCELLARI et al., 2003). Em seres humanos há relato de desenvolvimento de granuloma por corpo estranho causado pelo alumínio injetado (FAWCETT; SMITH, 1984).

A ocorrência de sarcoma de aplicação felino é relatada com mais freqüência pelos Clínicos Veterinários. Apesar de sua baixa prevalência, 
o tratamento, na maioria das vezes paliativo, e sua agressividade justificam maiores estudos e a compilação de informações relevantes, dando subsídios para que Médicos Veterinários optem pela melhor conduta, tanto para o tratamento quanto para a prevenção.

Portanto, o presente artigo tem como propósito fazer uma revisão sobre a doença, enfocando aspectos da epidemiologia, etiopatogenia, morfologia, abordagem clínica e cirúrgica, com intuito de fornecer aos colegas profissionais informações para a prevenção do problema e que possibilitem optar pela conduta mais adequada frente a essa afecção.

Epidemiologia, Etiopatogenia e Morfologia

Nos últimos 20 anos, têm-se observado aumento no número de relatos de sarcoma de tecidos moles em locais comumente utilizados para aplicação de medicações injetável em gatos. Embora seja conhecida a correlação entre a aplicação de vacinas e o desenvolvimento de sarcomas, a verdadeira incidência e a etiologia permanecem desconhecidas (SÉGUIN, 2002). Withrow e Macewen (2001) publicaram que qualquer elemento poderia ser responsável pelo aparecimento do sarcoma de aplicação em felinos. Autores de estudos epidemiológicos relatam uma incidência de sarcoma pós-vacinal em 0,63 a 3 sarcomas para cada 10000 felinos vacinados e 0,32 sarcomas para cada 10000 doses do total de vacinas administradas (GOBAR; KASS, 2002; SÉGUIN, 2002). A baixa prevalência dessa neoplasia em relação à quantidade de vacinas administradas sugere fatores inerentes a cada animal (NAMBIAR et al., 2001)

Em 2002 dois autores demonstraram que as vacinas com adjuvante são mais propensas a causar reação inflamatória do que aquelas sem adjuvante (GOBAR; KASS, 2002). Estudos epidemiológicos realizados no Havaí entre janeiro 1991 e maio 1992, estabeleceram relação causal clara entre as vacinações e o desenvolvimento subseqüente de tumor. Foram considerados quatro tipos de vacinas: a tríplice felina (rinotraqueíte- calicivirose e panleucopenia), FeLV, raiva e quádrupla felina (tríplice + clamidiose). Dentre estas vacinas a que foi fortemente associada com o surgimento de sarcoma em felinos foi a da FeLV em primeiro lugar, seguido da anti-rábica (WEIGAND; BREWER, 1996). Neste estudo demonstrou-se que uma única vacinação no espaço inter-escapular, local onde estas vacinas são aplicadas tradicionalmente, o risco de desenvolvimento de tumor aumentou para $50 \%$. Duas vacinações simultâneas aumentaram o risco para $127 \%$, enquanto que três ou mais vacinações resultaram em aumento no risco de 175\%. O período de latência de desenvolvimento do sarcoma de aplicação varia de três meses a três anos (KASS et al, 1993).

O SAF é caracterizado clinicamente pelo aparecimento de um nódulo solitário firme ou massa difusa, aderido a planos profundos e em região onde foi previamente administrada a vacina ou o fármaco (HENRY, 2001; OGILVIE; MOORE, 2002; WITHROW; VAIL, 2007). Segundo a VaccineAssociated Feline Sarcoma Task Force (VAFSTF) qualquer aumento de volume no local da aplicação após um mês, que tenha mais que dois centímetros de diâmetro ou que esteja presente por mais de três meses deve ser considerado como possibilidade de sarcoma de aplicação (HENRY, 2001).

Segundo a classificação histológica de tumores de tecidos moles e mesenquimais da pele de animais domésticos da Organização Mundial da Saúde (HENDRICK et al., 1998), o sarcoma de aplicação caracteriza-se histopatologicamente por hipercromatismo nuclear, formas multinucleadas e anaplasia. Para Madewell et al. (2001) o SAF tem características histológicas únicas, quando comparado ao sarcoma de não-aplicação. Apresenta inflamação predominantemente linfóide do tipo T, vascularização periférica abundante, variação na distribuição de células miofibroblasticas e células gigantes multinucleadas (COUTO et al., 2002; MADEWELL et al., 2001). Os macrófagos podem conter em seu citoplasma uma substância cinzaazulada que análises através de raios-X identificaram 
como alumínio (adjuvante) e oxigênio (OGILVIE; MOORE, 2002). Esses macrófagos contendo, possivelmente, material do adjuvante contido nas vacinas não são sempre observados, porém cavitações centrais, conseqüentes de paniculite granulosa necrotizante induzida por aplicações prévias são freqüentemente observadas (COUTO et al., 2002). O tipo mais freqüente é o fibrossarcoma, mas encontra-se também o rabdomiossarcoma, o mixossarcoma, o sarcoma indiferenciado e o fibrohistiocitoma maligno (COUTO et al., 2002; HENDRICK et al., 1994)

COUTO et al. (2002) afirmam que as correlações entre a presença de linfócitos $\mathrm{T}$ e/ou B no sarcoma de aplicação, o desenvolvimento tumoral e a resposta ao tratamento necessitam de mais estudos. Outro componente importante relatado no sarcoma vacinal foi a presença de células miofibroblásticas (MADEWELL et al., 2001). Essas, ativadas pelo tumor, formam uma cápsula que envolve os nódulos tumorais, prevenindo a penetração mecânica de linfócitos $\mathrm{T}$ e macrófagos, o que explica a presença destes agregados na periferia do tumor (COUTO et al., 2002; MADEWELL et al., 2001). Em seres humanos a presença desse tipo celular está relacionada com prognóstico favorável, enquanto que nos sarcomas vacinais isso não acontece (COUTO et al., 2002). A relação entre os miofibroblastos, os fibroblastos e as células da musculatura lisa presentes no sarcoma vacinal ainda é desconhecida (MADEWELL et al., 2001).

Os sarcomas de aplicação ocorrem mais comumente no subcutâneo, podendo se estender para a musculatura, enquanto os sarcomas de nãoaplicação ocorrem com maior freqüência na derme. Na maioria das vezes, se aderem a planos profundos, promovendo invasão profusa dos tecidos adjacentes com projeções digitiformes. Estudos descrevem o sarcoma de aplicação como mais agressivo em relação aos sarcomas não relacionados à aplicação, já que possuem alta taxa de crescimento, invadem profundamente os tecidos adjacentes, até mesmo ósseos, são mais metastáticos e a taxa de recidiva pós-cirurgia é $62 \%$ maior (SÉGUIN, 2002; POIRIER et al., 2002; WILLIAMS et al., 2001).

As razões específicas para a gênese neoplásica em locais de aplicação ainda não foram elucidadas. Os sarcomas felinos associados à aplicação são normalmente envolvidos por infiltrado inflamatório e o seu desenvolvimento pode ser precedido por uma resposta inflamatória exacerbada no local da aplicação (KASS et al., 1993; HENDRICK et al., 1994). Existem atualmente duas teorias sobre o surgimento do sarcoma de aplicação. Uma implica na composição do adjuvante das vacinas (alumínio), e outra envolve a deposição de altas concentrações de antígeno no tecido subcutâneo. O adjuvante, outros componentes da vacina, ou um fármaco, como lufenuron ou penicilina, pode causar uma resposta inflamatória local exacerbada, persistente, que perdura por mais de um mês, resultando em proliferação de fibroblastos e miofibroblastos, que podem sofrer alterações neoplásicas (WEIGAND; BREWER, 1996). Em algumas circunstâncias, sugere-se que o desenvolvimento acelerado de sarcomas no local de aplicação seja compatível com o envolvimento de oncogenes virais. Porém, o vírus da leucemia felina não foi detectado em 130 amostras de tumores avaliadas pela técnica de imuno-histoquímica e reação de polimerase em cadeia (PCR) (ELLIS et al., 1996).

Já está descrita a relação entre o desenvolvimento do sarcoma vacinal e algumas proteínas reguladoras da proliferação e do crescimento como p53, fator de crescimento de fibroblasto (FGF-b) e fator de crescimento transformador- $\alpha$ (TGF- $\alpha$ ) (NIETO et al., 2003; HERSHEY et al., 2005). Identificouse, por imuno-histoquímica, grande quantidade de receptores para fatores de crescimento em sarcoma de aplicação, como: o fator de crescimento derivado de plaqueta - PDFG, o fator de crescimento epidermal - EGF e o fator de crescimento transformador- $\beta$ TGF- $\beta$ (NIETO et al., 2003) Isso não foi observado ou obteve-se resposta fraca em sarcomas não relacionados à aplicação. (HENDRICK, 1998). Em um estudo sugeriu-se que a mutação no gene p53 
é uma das vias para o surgimento do sarcoma de aplicação (NAMBIAR et al., 2001).

\section{Abordagem Clínica e Cirúrgica}

\section{Diagnóstico}

Quando se suspeita de sarcoma de aplicação deve-se inicialmente elaborar o diagnóstico definitivo. Para o diagnóstico são utilizadas a citologia ou a biópsia. Segundo Hauck (2003), a biópsia é necessária para o diagnóstico definitivo, já que o grau de inflamação associado a esse tipo neoplásico faz com que o diagnóstico citológico se torne difícil. Chalita et al., 2002, demonstrou que a técnica citológica, na avaliação de tumores em pele e partes moles, mostrou-se eficiente para detectar malignidade e histogênese da afecção. Biópsia profunda da massa anormal é suficiente para o exame histopatológico definitivo, desde que não comprometa a realização do procedimento cirúrgico em seguida (NOVOSAD, 2003).

A possibilidade de sarcoma de aplicação deve ser considerada quando uma massa de qualquer tamanho persistir no sítio de aplicação por mais de três meses, quando for maior que $2 \mathrm{~cm}$, independente do tempo decorrido em relação à aplicação da vacina, ou quando ela aumentar de tamanho um mês após a aplicação vacinal.

\section{Exames complementares}

Hemograma completo, bioquímica sérica, urinálise e sorologia para o vírus da imunodeficiência felina (FIV) e para FeLV determinam o estado de saúde do paciente, bem como o provável prognóstico. Radiografias torácicas devem ser realizadas para investigar a possibilidade de metástases nos pulmões, que ocorrem em cerca de $10 \%$ a $24 \%$ dos casos. Linfonodos regionais devem ser avaliados por meio de palpação, radiografia, ultra-sonografia ou citologia conforme a necessidade. As metástases ocorrem inicialmente nos pulmões, mas já foram observadas em outros sítios, como linfonodos regionais, mediastino, pericárdio, fígado e pelve (SÉGUIN, 2002).

A utilização de tomografia computadorizada e/ ou ressonância magnética é extremamente útil para o planejamento da ressecção cirúrgica do tumor e para detectar possíveis metástases, que podem passar despercebidas nos métodos tradicionais de radiografia e ultra-sonografia (SÉGUIN, 2002). O exame físico, a palpação da neoplasia, deve ser realizado, porémpara cálculo da extensão e dovolume tumoral deve-se realizar exames complementares de imagem como a tomografia computadorizada. McENTEE e Samii (2000) estudaram 35 gatos com sarcomas vacinais, os quais tiveram seus volumes tumorais medidos por meio do exame físico e da tomografia computadorizada, com e sem contraste. Comparando as avaliações tumorais obtidas pelo exame físico e pela tomografia computadorizada concluíram que as imagens ofereceram a melhor indicação da extensão tumoral. Avaliando com exatidão a extensão da neoplasia, pode-se prescrever o melhor tratamento: excisão cirúrgica, radioterapia, quimioterapia, imunoterapia, terapia gênica ou uma combinação de duas ou mais (HAUCK, 2003).

\section{Tratamento cirúrgico}

A excisão cirúrgica é o principal tratamento para sarcomas de tecidos moles, de aplicação ou não (OGILVIE; MOORE, 2002; MCENTEE; PAGE, 2001). Devido à extensa infiltração e invasão de tecidos adjacentes, é necessário que se faça a ressecção ampla e profunda nas margens dos tecidos normais em todos os planos cirúrgicos ao redor da massa palpável. Em seres humanos e em animais maiores, como cães, uma excisão radical ou em bloco é freqüentemente possível, enquanto que nos gatos isso não ocorre, exceto aqueles tumores localizados na porção distal de membros ou em extremidades que podem ser amputadas (OGILVIE; MOORE, 2002). 
A primeira excisão cirúrgica deve ser definitiva e realizada com ampla margem, de pelo menos $3 \mathrm{~cm}$, incluindo ossos, músculos e outras estruturas que façam contato direto com a neoplasia e que entrem no planejamento das margens. Por exemplo, um gato com SAF em espaço interescapular requer excisão extensa, com pelo menos de $3 \mathrm{~cm}$ de margens do tecido normal incluindo o processo dorsal e o processo espinhoso da escápula. Deve-se realizar a remoção em bloco do tecido acometido sem contado direto com a massa tumoral (OGILVIE; MOORE, 2002; SÉGUIN, 2002). Após a excisão, toda a peça retirada em bloco deve ser encaminhada ao exame histopatológico para avaliar principalmente as margens cirúrgicas (SÉGUIN, 2002).

Em estudo retrospectivo, a taxa de recidiva estimada foi maior que $60 \%$ e $86 \%$ delas ocorreram em menos de seis meses após a excisão do tumor primário. Na realidade, o intervalo médio livre de tumor depois da ressecção referido por veterinários é de apenas 66 dias (HERSHEY et al., 2000). Já se sabe que na realização de cirurgia somente, mesmo nas mais extensas e agressivas, há chance de recidiva em 30\% a 70\% dos casos (HAUCK, 2003; MACY; COUTO, 2001). A detecção precoce e remoção cirúrgica radical do tumor têm prolongado o intervalo livre da doença e reduzido a taxa de recidiva (HERSHEY et al., 2000). Entretanto, o sarcoma felino associado à aplicação pode crescer rapidamente e freqüentemente se desenvolver em áreas que impossibilitam uma excisão completa. Em estudo com gatos tratados pela excisão cirúrgica a taxa de recidiva foi de $80 \%$ no tempo médio de quatro meses após a excisão cirúrgica. Somente $10 \%$ dos gatos se tornaram livres do tumor pelo período de um ano após a excisão (BREGAZZI et al., 2001). Aliado a um prognóstico ruim, a taxa global de metástases relacionada com o SAF pode ser em torno de $22,5 \%$, embora lesões distantes raramente sejam detectadas no momento do diagnóstico inicial (HERSHEY et al., 2000).

As cirurgias com margens incompletas resultam em até $70 \%$ de recidiva (COHEN et al., 2001;
MCENTEE; PAGE, 2001). Tumores que envolvem os membros recidivam com freqüência após a excisão local, mas a probabilidade de controle em longo prazo seguindo a amputação é alta. Da mesma forma, tumores da pina e membrana nictitante podem ser removidos com ampla margem de segurança. A hemipelvectomia deve ser realizada em tumores extensos dos membros, que comprometam a pelve. $\mathrm{Se}$ as margens ficarem comprometidas, a recidiva deve ser esperada (OGILVIE; MOORE, 2002). Tumores localizados no tórax ou no flanco requerem excisão ampla incluindo a remoção de costelas ou parede abdominal e utilização de malha de propileno. Mesmo com procedimentos cirúrgicos agressivos e com tentativas de reconstrução a recidiva ainda pode ocorrer (OGILVIE; MOORE, 2002; SÉGUIN, 2002; WITHROW; VAIL,2007).

\section{Radioterapia}

A radioterapia isolada não deve ser utilizada como modalidade única de tratamento para sarcomas associados à aplicação, podendo ser considerada como terapia paliativa (HAUCK, 2003; OGILVIE; MOORE, 2002; MCENTEE; PAGE, 2001). Os resultados mais promissores alcançados no tratamento de gatos com sarcomas associados à aplicação é a utilização de múltiplas terapias, incluindo radioterapia e cirurgia. Diversas modalidades de radioterapia foram avaliadas em combinação com a cirurgia, incluindo ortovoltagem, braquiterapia e megavoltagem (HAUCK, 2003). Em um estudo retrospectivo de 25 gatos tratados com cirurgia e radioterapia com megavoltagem, com ou sem quimioterapia associada, relatou-se sobrevida média de 701 dias, considerada como tempo livre do tumor. Destes, 7 foram submetidos à cirurgia seguidos da radioterapia e 18 foram tratados com a cirurgia, radioterapia e quimioterapia adjuvante (BREGAZZI et al., 2001).

Para avaliar o efeito da extensão da cirurgia foi realizado um estudo retrospectivo de 78 gatos, sendo que dezenove deles foram tratados com 
ressecção conservativa da massa tumoral (COHEN et al., 2001). Todos receberam radioterapia pósoperatória. O tempo médio livre da doença foi de 405 dias, com sobrevida média de 469 desde o começo do tratamento. Não houve diferença na recidiva entre os gatos tratados com a cirurgia conservativa e os tratados com ampla excisão cirúrgica. Os gatos que foram submetidos a múltiplas cirurgias antes da radioterapia tiveram diminuição do tempo livre do tumor quando comparados àqueles com uma única excisão cirúrgica (COHEN et al., 2001). Em estudo no qual a radioterapia foi administrada antes da excisão cirúrgica em 33 gatos, o intervalo médio livre da doença foi maior que 23 meses (CRONIN et al., 1998).

\section{Quimioterapia}

O impacto da quimioterapia no tratamento de sarcomas vacinais já está claramente identificado, porém seus efeitos são variados (HAUCK, 2003; NOVOSAD, 2003). A quimioterapia não deve ser considerada como tratamento definitivo, assim como a radioterapia deve ser utilizada como tratamento paliativo. Os sarcomas de tecido moles são tidos como pobremente responsivos. No entanto, a quimioterapia pode ser benéfica para gatos com tumores incompletamente excisados, recidivantes, tumores não cirúrgicos, ou para aqueles com doença metastática (WILLIAMS et al., 2001). Como tratamento adjuvante, a quimioterapia deve ser considerada importante para a associação entre terapias (SEGUIN, 2002). A utilização de diversos tipos de protocolos quimioterápicos resultou em resposta parcial, com mais de $50 \%$ de redução no tamanho tumoral inicial, e, em alguns casos, até remissão total do tumor, embora este fenômeno seja pouco freqüente (McENTEE; PAGE, 2001; HAUCK, 2003; HENRY, 2001; MACY; COUTO, 2001). Em estudo recente, em que se comparou o procedimento cirúrgico isolado ou com a quimioterapia pósoperatória associada, não se observou diferença na taxa de recidiva e de sobrevida entre os dois grupos. Porém, para o grupo de cirurgia seguido de quimioterapia foram selecionados os tumores de maior volume, sugerindo vício de amostragem (MARTANO et al., 2005).

A quimioterapia no período pré-operatório pode reduzir o tamanho da formação tumoral, facilitando a ressecção cirúrgica ou mesmo potencializando a sensibilidade do tumor à radiação. A quimioterapia pós-operatória tem o intuito de aumentar o tempo livre de doença, eliminando possíveis células neoplásicas remanescentes ou metastáticas. Dentre os agentes quimioterápicos que podem ser utilizados clinicamente estão a doxorrubicina, ciclofosfamida, carboplatina, mitoxantrone e vincristina (MCENTEE; PAGE, 2001).

A utilização de doxorrubicina aumenta o tempo para recidiva, porém não o tempo de sobrevida (POIRIER et al., 2002). A doxorubicina não interferiu no tempo livre do tumor em um estudo no qual se realizou procedimento cirúrgico associado à radioterapia pós-cirurgica, com ou sem doxorrubicina como quimioterapia adjuvante. $\mathrm{O}$ pequeno número de animais estudados e a natureza do estudo dificultaram determinar o verdadeiro impacto da quimioterapia (BREGAZZI et al., 2001). Estes resultados contrastam com os apresentados por King et al. (2001) com 61 gatos tratados com radioterapia pré-operatória e excisão cirúrgica com ou sem doxorubicina adjuvante. O tempo livre da doença nos gatos que receberam a droga foi mais prolongado quando comparado com os animais que não a receberam (média de 360 dias versus 162 dias). Katayama et al. (2004) observaram aumento na sensibilidade dos sarcomas vacinais à quimioterapia, baseada na doxorrubicina, quando utilizada juntamente com o mesilato de imatinibe. Quando o imatinibe foi utilizado no estudo sugeriuse que este inibe a ativação da tirosina-quinase nos sarcomas vacinais in vitro e inibe o crescimento tumoral em modelo de transplante xenográfico.

Em estudo avaliando a combinação de doxorrubicina e ciclofosfamida em gatos com 
tumores não resseccionados, metade apresentou redução de $50 \%$ ou mais da massa tumoral e $17 \%$ tiveram resolução de todo tumor detectado clinicamente. A sobrevida média de duração de resposta foi de 242 dias versus 83 dias do grupo não tratado (BARBER et al., 2000). Em estudo de fase I, sobre o uso de carboplatina em gatos com sarcoma vacinal, foi evidenciada ao menos resposta parcial, mais de 50\% de redução no tamanho tumoral inicial (HAUCK, 2003).

Autores indicaram o uso da doxorrubicina lipossomo-encapsulada (Doxil $\left.{ }^{\circledR}\right)$ (POIRIER et al., 2002; THAMMetal., 1997). Afase Idetriagem clinica da utilização de Doxil@, em animais com sarcoma vacinal, demonstrou resposta em 5 gatos de 13 tratados (THAMM et al., 1997). Thamm et al. (1997) demonstraram que o intervalo livre de progressão tumoral de animais que receberam ambas as formas de doxorrubicina, encapsulada e não-encapsulada, teve aumento significativo em comparação com os tratados somente com cirurgia. Em relação ao "intervalo livre de progressão" nenhuma diferença entre as formulações de doxorrubicina foi notada (POIRIER et al., 2002). A eficácia da doxorrubicina encapsulada em comparação a não encapsulada foi avaliada em estudo randômico (POIRIER et al., 2002). Foi observada toxicidade renal da formulação encapsulada de doxorrubicina, levando os pesquisadores à diminuição da dosagem (de $1,5 \mathrm{mg} / \mathrm{kg}$ para $1 \mathrm{mg} / \mathrm{kg}$ ) ou ao uso da doxorrubicina não encapsulada (POIRIER et al., 2002; THAMM et al., 1997).

No Brasil, a quimioterapia pode e deve ser indicada como tratamento, no pré ou pós-cirúrgico, pois a radioterapia ainda não é viável. A quimioterapia para pacientes felinos já está bem descrita e determinada, bem como seus efeitos colaterais.

A experiência coma utilização de quimioterápicos demonstra que a realização de quatro ciclos, a cada 21 dias, de quimioterapia a base de doxorrubicina, na dosagem de $1,5 \mathrm{mg} / \mathrm{kg}$ é tolerada pelos pacientes felinos, bem como tem efeito benéfico para a maioria dos animais. Não há a mesma experiência na utilização de outros protocolos quimioterápicos seguros e promissores como a carboplatina (CARNEIRO, 2006; POIRIER et al., 2002; THAMM et al., 1997).

\section{Imunoterapia}

A imunoterapia tem recebido pouca atenção (SEGUIN, 2002). A utilização de interleucina-2, produzida por células VERO implantadas nos pacientes com sarcoma de aplicação, após a intervenção cirúrgica, seguida de várias aplicações durante um período de dois meses resultaram em dois animais com recidiva local, três com metástases e sobrevida média de 16 meses (grupo com 16 felinos). Enquanto no grupo sem tratamento imunoterápico os pacientes apresentaram sobrevida média de oito meses e um deles apresentou anafilaxia (QUINTINCOLONNA et al., 1996). Apesar de promissores resultados, a imunoterapia ainda não é utilizada na rotina para tratamento de sarcomas de aplicação no Brasil e no mundo. Isso se deve ao baixo interesse comercial e custo ainda elevado da técnica.

\section{Prognóstico}

Alguns fatores de prognósticos seguidos à excisão cirúrgica foram estudados, dentre eles: localização do tumor, cirurgia com as margens livres ou comprometidas, cirurgias anteriores à ressecção definitiva e marcadores moleculares (COUTO et al., 2002; HERSHEY et al., 2000; HERSHEY et al., 2005; MCENTEE; SAMII, 2000; MARTANO et al., 2005). Em estudo com 35 gatos realizado por Ogilvie e Moore (2002), a localização do tumor não teve nenhuma relação com a recidiva, já nos gatos que possuíam margens cirúrgicas livres de células neoplásicas, o tempo médio livre do tumor foi de 16 meses e naqueles com margens cirúrgicas comprometidas a recidiva ocorreu no tempo médio de 4 meses. Igualmente, aqueles gatos que não foram submetidos a cirurgias prévias à cirurgia definitiva 
possuíam um tempo livre do tumor superior a 16 meses comparados com 5 meses naqueles que foram submetidos a cirurgias anteriores à definitiva. Avaliando o prognóstico seguido da excisão cirúrgica isolada, Hershey et al. (2000) encontraram sobrevida média de 576 dias. Observaram também que houve diferença significativa no tempo da primeira recidiva entre os gatos tratados por ampla excisão versus os tratados por excisão marginal, 419 dias versus 66 dias, respectivamente.

Hershey et al. (2005), demonstraram que, animais em que as células tumorais exibiam p53 no núcleo, tinham tempo maior para o aparecimento de recidiva (325 dias) do que os que apresentavam p53 citoplasmática (135 dias). Entretanto, não foi observada diferença significativa no aumento da sobrevida. É observado em seres humanos, que a expressão citoplasmática de p53 está associada com um prognóstico pior (HERSHEY et al., 2000). Nesse mesmo trabalho também observaram que as margens tumorais que eram histologicamente livres de alterações apresentavam marcação para o p53, caracterizando o processo como "field caracterization", o que leva à hipótese de lesões précancerosas ou extensões do tumor primário.

\section{Prevenção}

Considerando-se que o SAF é altamente agressivo, de difícil tratamento e de prognóstico ruim, deve-se proceder como objetivo de diminuir sua incidência e sua morbidade. Isso pode ser alcançado por meio da implantação de medidas que previnam o aparecimento dessa neoplasia.

Inicialmente deve-se diminuir a utilização de aplicações injetáveis em felinos, reservar vias injetáveis para casos em que esta não pode ser evitada e dar prioridade para a via subcutânea, pois a formação da massa é mais facilmente observada pelo proprietário (MACY; COUTO, 2001, NOVOSAD, 2003). As aplicações devem ser evitadas principalmente em animais que já possuem relato de desenvolvimento de reação inflamatória exacerbada e/ou sarcoma (SÉGUIN, 2002).

Com relação à vacinação devemos ponderar sobre vários aspectos, como quais patógenos aquele animal está realmente sendo exposto e se esse patógeno envolvido promove uma zoonose (SMITH, 1995). A VAFSTF sugere, nos EUA que a vacinação seja realizada a cada 3 anos, porém no Brasil temos patógenos endêmicos, como o da raiva que nos impedem de fazer esse intervalo. Em gatis temos o problema da leucemia felina. Além disso, a vacinação anual anti-rábica é obrigatória em todo o território nacional. Ainda recomenda-se a utilização de vacinas atenuadas sem adjuvantes ou as mortas com adjuvantes que não causem reação inflamatória exuberante(HENRY, 2001; MACY; COUTO, 2001). A VAFSTF recomenda mudança e padronização das aplicações injetáveis em felinos, devendo evitarse a região interescapular. As vacinas anti-rábicas devem ser aplicadas em membro pélvico direito, as contra o vírus da leucemia felina no membro pélvico esquerdo e outras aplicações em membro torácico direito, sendo que estas aplicações devem ser realizadas mais distalmente possível. Macy e Couto (2001) atentam para a utilização do mesmo local para múltiplas aplicações injetáveis predispor ao aparecimento do SAF.

O Médico Veterinário tem o dever de informar o proprietário sobre os riscos do desenvolvimento do SAF. Deve-se observar individualmente cada paciente e esclarecer para o proprietário as reais possibilidades do desenvolvimento de doenças, prevenidas por vacinações, em relação à possibilidade do desenvolvimento do sarcoma de aplicação. Caso as vacinações anuais não sejam aplicadas, enfermidades que levam a quadros de morbidade ou até a morte podem voltar a apresentar alta incidência, bem como o desencadeamento de problemas de saúde publica, quando se trata de vacinação contra zoonoses.

A vacinação é questionada pelos proprietários e porMédicos Veterinários, porémessequestionamento deve ser embasado em informações da literatura, 
com o objetivo de saúde para o paciente felino e também para o ser humano. Assim conseguir evitar o surgimento de afecções já erradicadas ou de baixa prevalência em felinos e o aumento de casos de zoonoses em seres humanos, como o ocorrido nos EUA (RASSNICK, 2006).

\section{Conclusão}

O sarcoma de aplicação felino é um tumor extremamente agressivo que necessita de diagnóstico e tratamento precoces. O diagnóstico pode ser realizado por exame citológico ou por biopsia. A tomografia computadorizada oferece grande contribuição na determinação da extensão do tumor para melhor planejamento cirúrgico. A excisão cirúrgica com ampla margem de segurança pode ser eficaz, mas a melhor abordagem parece ser a multimodalidade de terapias (cirurgia/quimioterapia/ radioterapia). Mesmo utilizando a combinação de terapias as taxas de recidiva são elevadas.

\section{Agradecimentos}

À Fundação de Amparo à Pesquisa do Estado de São Paulo (FAPESP), pela bolsa de mestrado para Carolina Scarpa Carneiro.

\section{Referências}

BARBER, L. G.; SORENMO, K. U.; CRONIN, K. L.; SHOFER, F. S. Combined doxorubicin and cyclophosphamide chemotherapy for nonresectable feline fibrosarcoma. Journal of the American Animal Hospital Association, Lakewood, v.36, n.5, p.416-421, 2000.

BREARLEY, M. J. Vaccine-associated feline sarcoma. Veterinary Record, Letters, v.152, n.15, p.478, 2003.

BREGAZZI, V. S.; LaRUE, S. M.; McNIEL, E.; MACY, D. W.; DERNELL, W. S.; POWERS, B. E.; WITHROW, S. J. Treatment with a combination of doxorubicin, surgery, and radiation versus surgery and radiation alone for cats with vaccine-associated sarcomas: 25 cases (1995-2000). Journal of American Veterinary Medical Association, Schaumburg, v.218, n.4, p.547-550, 2001.
BURACCO, P.; MARTANO, M.; MORELLO, E.; RATTO, A. Vaccine associated-like fibrosarcoma in the site of a deep nonabsorbable suture in a cat. Veterinary Journal, London, v.163, n.1, p.150-107, 2002.

CARNEIRO, C. S. Estudo clínico sobre o efeito da administração de doxorrubicina no sarcoma de aplicação em felino. 2006. Dissertação (Mestrado em Clínica Cirúrgica Veterinária) - Faculdade de Medicina Veterinária e Zootécnica, Universidade de São Paulo, São Paulo.

CHALITA, M. C. C.; MATERA, J. M.; ALVES, M. T. S.; LONGATTO FILHO, A. Tumores em pele e partes moles de cães: estudo clínico e cito-histológico. Revista de Educação Continuada do CRMV-SP, São Paulo, v.5, n.2, p.171-180, 2002.

COHEN, M.;WRIGHT, J. C.; BRAWNER, W. R.; SMITH, A. N.; HENDERSON, R.; BEHREND, E. N. Use of a surgery and electron beam irradiation, with or without chemotherapy, for treatment of vaccineassociated sarcomas in cats: 78 cases (1996-2000). Journal of American Veterinary Medical Association, Schaumburg, v.219, n.11, p.1582-1589, 2001.

COUTO, S. S.; GRIFFEY, S. M.; DUARTE, P. C.; MADEWELL, R. Feline vaccine-associated fibrosarcoma: morphologic distinctions. Veterinary Pathology, Washington, v.39, p.33-41, 2002.

CRONIN, K.; RODNEY, L.; SPODNICK, G.; DODGE, R.; HARDIE, E. N.; SYLVESTER, P.; USCAMDER, D.; THRALL, D. E. Radiation therapy and surgery for fibrosarcomas in 33 cats. Veterinary Radiology and Ultrasound, Raleigh, v.39, n.1, p.51-56, 1998.

DUBIELZIG, R. R. Ocular sarcoma following trauma in three cats. Journal of American Veterinary Medical Association, Schaumburg, v.184, n.5, p.578-581, 1984.

ELLIS, J. A.; JACKSON, M. L.; BARTSCH, R. C.; McGILL, L. G.; MARTIN, K. M.; TRASK, B. R.; HAINES, D. M. Use of immunohistochemistry and polymerase chain reaction for detection of oncornaviruses in formalin-fixed, paraffin-embedded fibrossarcomas of cats. Journal of American Veterinary Medical Association, Schaumburg, v.209, p.767-771, 1996.

FAWCETT, H. A.; SMITH, N. P. Injection-site granuloma due to aluminium. Archives of Dermatology, Chicago, v.120, p.1318-1322, 1984.

GOBAR, G. M.; KASS, P. H. World Wide Web-based survey of vaccination practices, postvaccinal reactions, and vaccine site-associated sarcomas in cats. Journal of American Veterinary Medical Association, Schaumburg, v.220, n.10, p.1477-1482, 2002. 
HAUCK, M. Feline injection site sarcomas. Veterinary Clinics Small North America Animal Practice, Philadelphia, v.33, n.3, p.553-571, 2003.

HENDRICK, M. J.; GOLDSCHMIDT, M. H. Do injection site reactions induce fibrosarcomas in cats? Journal of the American Veterinary Medical Association, Schaumburg, v.199, n.8, p.968, 1991.

HENDRICK, M. J.; SHOFER, F. S.; GOLDSCHMIDT, M. H.; HAVILAND, J. C.; SCHELLING, S. H. ENGLER, S. J.; GLIATTO, J. M. Comparison of fibrosarcomas that developed at vaccination sites and at nonvaccination sites in cats: 239 cases (1991-1992). Journal of the American Veterinary Medical Association, Schaumburg, v.205, n.10, p.1425-1429, 1994.

HENDRICK, M. J.; MAHATFEY, E.; MOORE, F.; VOS, J.; WALDER, E. Histological classification of mesenchymal tumors of skin and soft tissues of domestic animals. Washington: AFIP, 1998. v.2.

HENRY, C. J. Update on Vaccine-Associated Sarcomas. In: AUGUST, J. R. Consultations in feline internal medicine. Philadelphia: WB Saunders, 2001. v.4, p.541547.

HERSHEY, A. E.; DUBIELZIG, R. R.; PADILLA, M. L.; HELFAND, S. C. Aberrant p53 expression in feline vaccine-associated sarcomas and correlation with prognosis. Veterinary Pathology, Washington, v.42, n.6, p.805-811, 2005.

HERSHEY, A. E.; SORENMO, K. U.; HENDRICK, M. J.; SHOFER, F. S.; VAIL, D. M. Prognosis for presumed feline vaccine-associated sarcoma after excision: 61 cases (1986-1996). Journal of the American Veterinary Medical Association, Schaumburg, v.216, n.1, p.58-61, 2000.

KASS, P. H.; BARNES, W. G.; SPANGLER, W. L.; CHOMEL, B.; CULBERTSON, M. R. Epidemiologic evidence for a causal relation between vaccination and fibrosarcoma tumorigenesis in cat. Journal of the American Veterinary Medicine Association, Schaumburg, v.203, n.3, p.396-405, 1993.

KATAYAMA, R.; HUELSMEYER, M. K.; MARR, A. K.; KURZMAN, I. D.; THAMM, D. H.; VAIL, D. M. Imatinib mesylate inhibits platelet-derived growth factor activity and increases chemosensitivity in feline vaccine-associated sarcoma. Cancer Chemotherapy and Pharmacology, Berlin, v.54, n.1, p.25-33, 2004.

KING, G. K.; HARRIS, D. F.; HOTTINGER, H.; FREEMAN, K. P.; HAHN, K. A. Prolonged remission and survival time in cats with injection-site sarcomas using pre-operative radiation and doxorubicin protocol: 61 cases (1998-2000). American College Veterinary Radiology and Ultrasound, Raleigh, v.42, p.585, 2001
KIRPENSTEIJN, J. Feline injection site-associated sarcoma: Is it a reason to critically evaluate our vaccination policies? Veterinary Microbiology, Amsterdam, v.117, n.1, p.59-65, 2006.

MACY, D.W.; COUTO, C. G. Prevention and treatment of injection-site sarcomas. Journal of Feline Medicine and Surgery, London, v.3, n.4, p.169-170, 2001.

MADEWELL, B. R.; GRIFFEY, S. M.; McENTEE, M. C.; LEPPERT, V. J.; MUNN, J. R. Feline vaccineassociated fibrosarcoma: an ultrastructural study of 20 tumors (1996-1999). Veterinary Pathology, Washington, v.38, n.2, p.196-202, 2001.

MARTANO, M.; MORELLO, E.; UGHETTO, M.; IUSSICH, S.; PETTERINO, C.; CASCIO, P.; BURACCO, P. Surgery alone versus surgery and doxorubicin for the treatment of feline injection-site sarcomas: a report on 69 cases. Veterinary Journal, London, v.170, n.1, p.84-90, 2005.

McENTEE, M. C.; PAGE, R. L. Feline vaccine-associated sarcomas. Journal of Veterinary Internal Medicine, Lawrence, v.15, p.176-182, 2001.

McENTEE, M. C.; SAMII, V. F.; The utility of contrast enhanced tomography in feline vaccine associated sarcomas: 35 cases. Veterinary Radiology Ultrasound, Raleigh, v.41, p.575, 2000.

NAMBIAR, P. R.; JACKSON, M. L.; ELLIS, J. A.; CHELACK, B. J.; KIDNEY, D. M.; HAINES, D. M. Immunohistochemical detection of tumor suppressor gene p53 protein in feline vaccine site-associated sarcomas. Veterinary Pathology, Washington, v.38, p.236-238, 2001.

NIETO, A.; SANCHEZ, M. A.; MARTINEZ, E.; ROLLAN, E. Immunohistochemical expression of p53, fibroblast growth factor-b, and transforming growth factor- $\alpha$ in feline vaccine-associated sarcomas. Veterinary Pathology, Washington, v.40, p.651-658, 2003.

NOVOSAD, C. A. Principles of treatment for vaccineassociated sarcomas. Clinical Techniques in Small Animal Practice, Philadelphia, v.18, n.2, p.115-117, 2003.

OGILVIE, G. K.; MOORE, A. S. Feline oncology: a comprehensive guide to compassionate care. New Jersey: Veterinary Learning Systems, 2002.

POIRIER, V. J.; THAMM, D. H.; KURZMAN, I. D.; JEGLUM, K. A.; CHUN, R.; OBRADOVICH, J. E.;O'BRIEN, M.; FRED III, R. M.; PHILLIPS, B. S.; VAIL, D. M. Liposome-encapsulated doxorubicin (Doxil $\left.{ }^{\circledR}\right)$ e doxorubicin in treatment of vaccineassociated sarcoma in cats. Journal of the American Veterinary Medical Association, Schaumburg, v.16, n.6, p.726-731, 2002. 
QUINTIN-COLONNA, F.; DEVAUCHELLE, P.; FRADELIZI, D.; MOUROT, B.; FAURE, T.; KOURILSKY,P.; ROTH, C.; MEHTALI, M. Gene therapy of spontaneous canine melanoma and fibrosarcoma by intratumoral administration of histoincompatible cells expressing human interleukin-2. Gene Therapy, London, v.3, n.12, p.1104-1106, 1996.

RASSNICK, K. M. Feline vaccine-associated sarcomas: the problem is not over yet. In: ANNUAL FORUM - AMERICAN COLLEGE OF VETERINARY INTERNAL MEDICINE, 24., 2006, New Orleans. Anais... Los Angeles, 2006.

SÉGUIN, B. Feline injection site sarcomas. Veterinary Clinics of North America Small Animal Practice, v.32, n.4, p.983-985, 2002.

SMITH, C. A. Are we vaccinating too much? Journal of the American Veterinary Medical Association, Schaumburg, v.207, n.4, p.421-425, 1995.

THAMM, D. H.; MACEWEN, E. G.; CHUN, R. Phase I clinical trial of Doxil ${ }$, a stealth liposome encapsulated doxorubicin, in cats with malignant tumor. AMERICAN COLLEGE OF VETERINARY RADIOLOGY COMBINED CONFERENCE, 1997, Chicago. Proceedings... Chicago, IL. Veterinary Cancer Society, 1997. p.38.
VASCELLARI, M.; MELCHIOTTI, E.; BOZZA, M. A.; MUTINELLI, F. Fibrosarcoma at presumed sites of injection in dogs: characteristics and comparison with nonvaccination site fibrosarcomas and feline postvaccinal fibrosarcomas. Journal of Veterinary Medicine. Series A, Physiology, Pathology, Clinical Medicine, Germany, v.50, p.286-291, 2003.

WEIGAND, C. M.; BREWER, W. G. Vaccination-site sarcomas in cats. Compendium on Continuing Education for the Practicing Veterinarian, Princeton, v.18, n.8, p.869-875, 1996.

WILLIAMS, L. E.; BANERJI, N.; KLAUSNER, J. S.; KAPUR, V.; KANJILAL, S. Establishment of two vaccine-associated feline sarcoma cell lines and determination of in vitro chemosensitivity to doxorubicin and mitoxantrone. American Journal of Veterinary Research, Schaumburg, v.62, n.9, p.1354-1357, 2001.

WITHROW, S. J.; MACEWEN, E. G. Small animal clinical oncology. 3ed. Philadelphia: WB Saunders Company, p. 283-304. 2001.

WITHROW, S. J.; VAIL, D. M. Withrow and MacEwen's small animal clinical oncology. 4.ed. Philadelphia: WB Saunders Company, 2007. 